\title{
High redshift ellipticals: prospects for the VLT
}

\author{
Andrea Cimatti ${ }^{1}$ \\ Osservatorio Astrofisico di Arcetri, Largo E. Fermi 5, I-50125, Firenze, Italy
}

\begin{abstract}
The results and the present limits of the observations of high- $z$ ellipticals are discussed in the framework of VLT imminent and future instruments.
\end{abstract}

\section{When and how did massive ellipticals form ?}

The formation of the present-day massive elliptical galaxies remains one of the most controversial issues of galaxy evolution and structure formation. In CDM hierarchical merging models (e.g. [1,2,3]), massive ellipticals form at relatively low redshift (e.g. $z<1$ ) through the merging of spiral galaxies. In such scenarios, massive, old and passively evolving ellipticals are extremely rare objects at $z>1$. In marked contrast, other scenarios suggested that massive ellipticals formed at higher redshifts (e.g. $z>2-3$ ) through an intense initial starburst event followed by pure luminosity evolution (PLE) of the stellar population to nowadays (e.g. [4, 5 , (6), thus implying a constant comoving number density of passively evolving ellipticals at $z \sim 0$ and $z>1$. In this scenario, a substantial number of extremely red objects (EROs) with the colors of an old stellar population at $z>1$ (e.g. $R-K>5.3$ ) and $r^{1 / 4}$ surface brightness profiles typical of dynamically relaxed spheroidals is expected to be found in near-IR selected (i.e. stellar mass selected) galaxy samples.

Imaging surveys with typical fields of $\sim 1-50 \operatorname{arcmin}^{2}$ provided very discrepant results. Some found a clear deficit of old ellipticals at $z \sim 1$ or $z>1$ compared to passive evolution models (e.g. [7, 8, 9, 10, 11, 13]), whereas others found evidence for a constant comoving density ([14, 15, 16, 17, 18, 19]). A large part of the above discrepancies is certainly due to the strong ERO angular clustering (i.e. field-to-field density variations) that was discovered thanks to wider field surveys (20,21]). The results of such surveys suggest that the observed angular clustering is the signal of the underlying $3 \mathrm{D}$ large scale structure of massive ellipticals[22], and showed that the surface density of $z>1$ passive elliptical candidates is consistent with that expected in PLE models, thus suggesting that most field ellipticals were fully assembled at least by $z \sim 2.5$ (23]). However, follow-up observations are needed to confirm that most EROs are passive ellipticals because it is known that some EROs are dust-reddened starburst galaxies, thus representing a "contamination" in color-selected samples of elliptical candidates at $1<z<2(24,25,26])$. 


\section{The results of VLT 1st generation instruments}

Fig. 1 shows the spectrum of a $z \sim 1.1$ passive elliptical as observed with the ESO VLT equipped with the optical imager-spectrograph FORS2 ([27]). No emission lines are present, and the main features are the $4000 \AA$ continuum break together with strong CaII H\&K absorptions and other weaker absorption lines. When such a spectrum is redshifted to $z>1.4$, the $4000 \AA$ and the CaII lines exit from the accessible optical spectral range and the redshift identification relies only on weak absorptions. Fig. 2 shows an example of an elliptical candidate at $z \sim 1.6$ that can be taken as a clear example of the difficulties in identifying the nature and the redshifts of high- $z$ passive ellipticals (see also [28,29]).

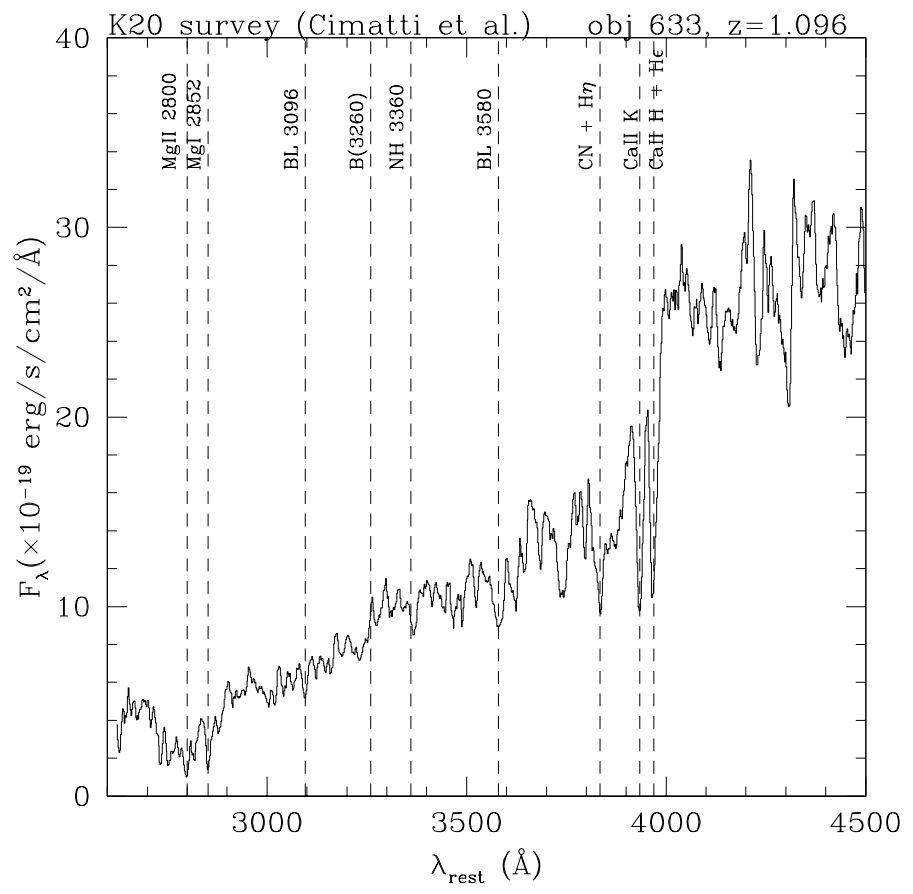

Fig. 1. ESO VLT+FORS2 spectrum of a $z \sim 1.1$ elliptical with $R \sim 23$ (from 27])

When elliptical candidates are too faint for optical spectroscopy and/or expected to be at $z>1.5$, the only possibility is to move to near-IR spectroscopy in order to search for the $4000 \AA$ break and the CaII H\&K lines redshifted at $\lambda_{o b s}>1 \mu \mathrm{m}$. However, the general faintness of the target continua makes such observations difficult and time-consuming, and the results of both Keck 
and VLT seeing-limited near-IR spectroscopy provided so far rather ambiguous results $([30,25,31])$.

Despite the difficulties of optical and near-IR spectroscopy, a substantial number of $z>1$ passively evolving ellipticals has been identified in recent surveys [33,28, 34, 27, 35, 29]. The inferred ages of the stellar populations $(\sim 2-5 \mathrm{Gyr})$ are consistent with such galaxies being formed at remote cosmological epochs. In addition, morphological studies based on HST imaging further confirmed the existence of a population of dynamically relaxed high- $z$ spheroids through the analysis of their surface brightness profiles (e.g. [32, 11, 12, 13].

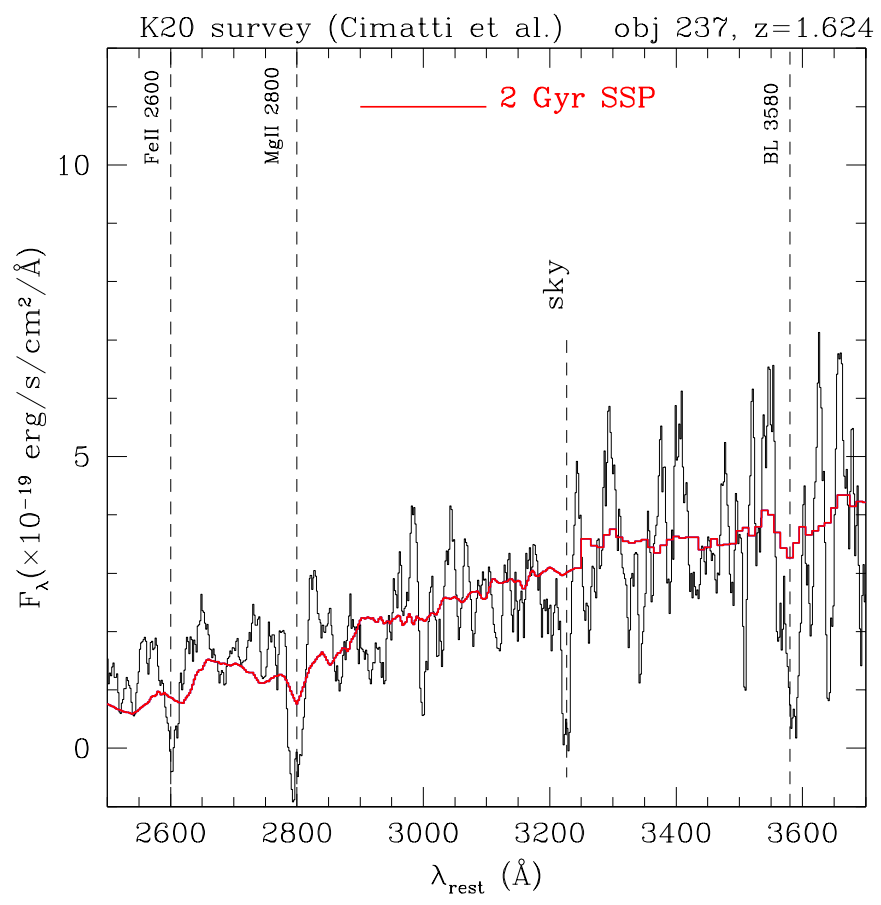

Fig. 2. ESO VLT+FORS2 spectrum of a $z \sim 1.6$ elliptical candidate with $R \sim 25.9$ and $R-K=6.8$ plotted together with a Bruzual \& Charlot 2 Gyr old SSP model (from [27]).

\section{$3 \quad$ Future prospects for the VLT}

Despite the successful observations of VLT, Keck and HST to uncover a substantial population of passively evolving ellipticals at $z>1$, many questions need still to be answered before drawing final conclusions on the formation and 
evolution of massive galaxies: what is the fraction of ellipticals as a function of $z$ and $K$ ? what are their masses, ages and redshifts of formation? what is the evolution of their luminosity function and clustering with respect to model predictions ? Addressing the above questions requires the use of different observing techniques, and three ideal VLT instruments could play a crucial role:

- AO-aided near-IR imager. Such an instrument, especially if aided by a laser guide star (LGS) system allowing a flexible pointing of the telescope (i.e. not limited to targets close to bright natural stars), would allow to study the morphology of elliptical candidates not only to confirm their nature by analysing their surface brightness profiles, but also to derive radial color gradients and to study the evolution of the fundamental plane (e.g. via the Kormendy relation) once the redshifts are known.

- AO-aided near-IR spectrograph. The scientific aim of such an instrument would be to spectroscopically confirm the nature of the passive elliptical candidates and to measure their redshifts. A simultaneous coverage of the near-IR spectral range $(Z J H K)$ would be essential to reduce the observation time and to overcome the problem of matching and inter-calibrating independent $Z, J, H, K$ spectra (e.g. ISAAC). A high throughput in the $Z$ and $J$ bands would be also crucial to work efficiently in the spectral range where the $4000 \AA$ break falls for $1.5<z<2.5$. A MOS capability would be obviously important to increase the multiplex. Such a spectrograph should also have a low spectral resolution mode (e.g. R 200-500) in order to derive, together with optical photometry, accurate continuum spectrophotometry for the faintest targets in order to estimate their "spectrophotometric" redshifts whenever it is impossible to measure their spectroscopic redshifts (e.g. Cimatti et al. 1999; Soifer et al. 1999). A moderately high spectral resolution would be important to estimate the masses of the brightest targets through the velocity dispersion of the absorption lines. It should be noted here that for faint galaxy spectroscopy (where the slit width cannot be much narrower than the size of the galaxy), low-order AO corrections are sufficient to provide a significant improvement of the $\mathrm{S} / \mathrm{N}$ ratio thanks to reduction of the sky background compared with seeing-limited spectroscopy made with typical slit widths of $1^{\prime \prime}$.

- Near-IR wide-field imager (e.g. $20 \times 20 \operatorname{arcmin}^{2}$ ). A VLT near-IR WFI with optimal seeing sampling (e.g. $0.15^{\prime \prime} /$ pixel) would be crucial to exploit the image quality of the VLT and to push the photometry to the limits of a 8mclass telescope. Such an instrument would allow to perform ultradeep surveys and to select and to study high- $z$ ellipticals beyond the spectroscopic limits. In this respect, it would be essential to have such an imager equipped with a set of medium-band filters in order to derive spectral energy distributions and $z_{\text {phot }}$ with a high level of accuracy. Such an instrument would play a key role in statistical studies such as the evolution of the luminosity function and of the clustering of ellipticals. 


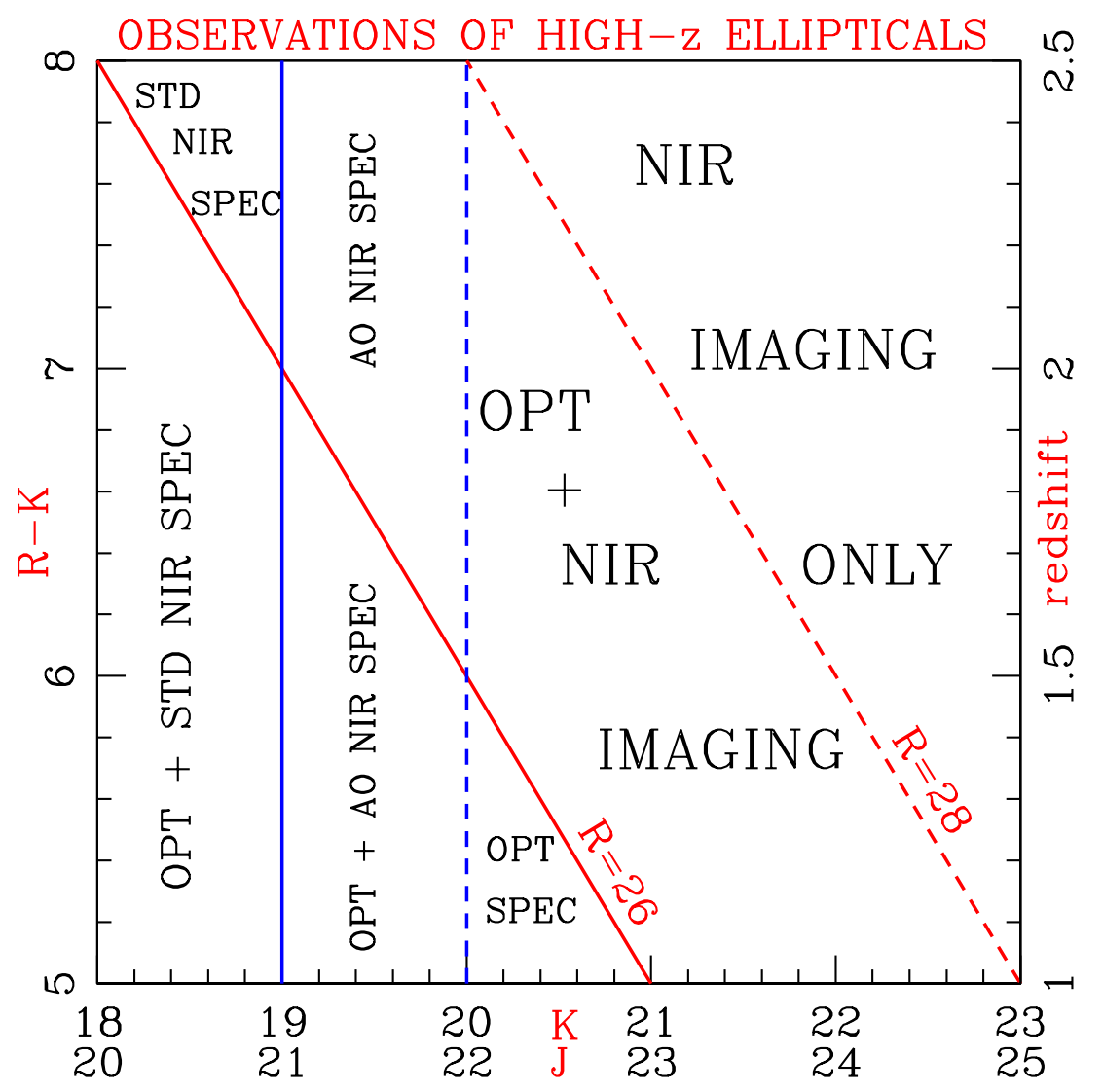

Fig. 3. The figure displays the different observing regimes for high- $z$ passive ellipticals as a function of their colors, magnitudes and redshifts. The $\mathrm{X}$-axis shows the $J$ and $K$ magnitudes, assuming a color of $J-K \sim 2$ which is characteristic of an old passively evolving system at $1<z<2$. The $\mathrm{Y}$-axes show the $R-K$ color and the redshift (the $R-$ $K$ color being a function of $z$ for $1<z<2$ ). The continuous and dashed diagonal lines indicate the location of $R=26$ and $R=28$ objects respectively. The vertical continuous line indicate the present limit of seeing-limited near-IR continuum spectroscopy with VLT+ISAAC. The vertical dashed line indicates a conservative limit of AO-aided near-IR continuum spectroscopy expected for the VLT. Different observing regimes can be envisaged. Continuum optical spectroscopy can be realistically performed down to $R \sim 26$. Near-IR spectroscopy without AO can be done only for relatively bright targets $(K \sim 19, J \sim 21)$ and a $\geq 1$ magnitude gain is expected with the aid of AO. For targets fainter than $K \sim 20$, only imaging can be performed. For targets with, say, $R<28$, optical and near-IR imaging can be done with reasonable integration times and photometric accuracy, but for galaxies with $R>28$ optical imaging becomes very difficult and most of the photometric information comes from near-IR observations only. 


\section{Summary}

A significant population of $z>1$ passively evolving elliptical candidates was unveiled thanks to recent wide-field imaging surveys. The confirmation of their nature and the measurement of their redshifts are very challenging even with 8$10 \mathrm{~m}$ class telescopes and the results obtained so far are limited to the brightest objects $(K<19)$. Much observational work can still be done with the 1st generation VLT instruments such as the red-upgraded FORS2 (optical imaging and spectroscopy), CONICA and SINFONI (AO-aided near-IR imaging and spectroscopy). An AO- or MCAO-aided near-IR imager-spectrograph (with MOS capability) and a near-IR WFI equipped with medium-band filters are the most desirable 2nd generation VLT instruments expected to play a crucial role in the understanding of the formation and evolution of the massive ellipticals.

\section{References}

1. Kauffmann G. 1996, MNRAS, 281, 487

2. Baugh C.M., Cole S., Frenk C.S. 1996, MNRAS, 283, 1361

3. Baugh C.M., Cole S., Frenk C.S., Lacey C.G. 1998,ApJ,498,504

4. Eggen, O. J.; Lynden-Bell, D.; Sandage, A. R. 1962, ApJ, 136, 748

5. Larson R.B. 1974, MNRAS, 166, 686

6. Arimoto N. \& Yoshii Y. 1987, A\&A, 173, 23

7. Zepf S.E. 1997, Nature, 390, 377

8. Franceschini A. et al. 1998, ApJ, 506, 600

9. Barger A.J. et al. 1999, AJ, 117, 102

10. Menanteau F. et al. 1999 MNRAS, 309, 208

11. Treu T., Stiavelli M. 1999, ApJ, 524, L27

12. Stiavelli M., Treu T. 2000, astro-ph/0010100

13. Rodighiero G. et al. 2001, MNRAS, in press (astro-ph/0101262)

14. Totani T., Yoshii J. 1997, ApJ, 501, L177

15. Benitez N. et al. 1999, ApJ, 515, L65

16. Broadhurst T.J., Bouwens R.J. 1999, ApJ, 530, L53

17. Schade D. et al. 1999, ApJ, 525, 31

18. Scodeggio M., Silva D.R. 2000, A\&A. 359. 953

19. Im M. et al. 2001, ApJ, in press (astro-ph/0011092

20. Daddi E. et al. 2000, A\&A. 361, 535

21. McCarthy P.J. et al. 2000, astro-ph/0011499

22. Daddi E. et al. 2001, A\&A, in press (astro-ph/0107340)

23. Daddi E., Cimatti A., Renzini A. 2000, A\&A, 362, L45

24. Cimatti A., Andreani P., Röttgering H., Tilanus R. 1998, Nature, 392, 895

25. Cimatti A. et al. 1999, A\&A, 352, L45

26. Dey A. et al. 1999, ApJ, 519, 610

27. Cimatti et al., in preparation (see http://www. arcetri.astro.it/ k20/)

28. Liu M.C. et al. 2000, AJ, 119, 2556

29. Stockton A. 2001, astro-ph/0104191

30. Soifer B.T. 1999, AJ, 118, 2065

31. Glassman T.M., Larkin J.E. 2000, ApJ, 539, 570

32. Moriondo G., Cimatti A., Daddi E. 2000, A\&A, in press

33. Spinrad H. et al. 1997, ApJ, 484, 581

34. Cohen J.G. et al. 1999. ApJ. 512. 30

35. Dunlop J.S. 1999, astro-ph/9912380 